\title{
Researching Hemispheric Specialization in the Control of Final Position Accuracy Using the Transcranial Direct Current Stimulation
}

\author{
Hemispheric Specialization and Final Position Accuracy \\ Mostafa Teymuri Kheravi ${ }^{1}$, Alireza Saberi Kakhki ${ }^{1}$, Hamidreza Taheri ${ }^{1}$, \\ Ali Ghanaie Chamanabad ${ }^{2} \&$ Mohammad Darainy $^{3}$ \\ ${ }^{1}$ Faculty of Physical Education and Sport Sciences, Ferdowsi University of Mashhad, Mashhad, Iran \\ ${ }^{2}$ Faculty of Psychology and Educational Sciences, Ferdowsi University of Mashhad, Mashhad, Iran \\ ${ }^{3}$ Department of Psychology, McGill University, Montreal, Quebec, Canada \\ Correspondence: Alireza Saberi Kakhki, Faculty of Physical Education and Sport Science, Ferdowsi University of \\ Mashhad, Mashhad, Iran. Tel: 98-915-168-9538.
}

Received: March 17, 2017 Accepted: April 14, 2017 Online Published: April 26, 2017

doi:10.5539/gjhs.v9n7p138 URL: https://doi.org/10.5539/gjhs.v9n7p138

\begin{abstract}
Transcranial Direct Current Simulation (tDCS) can improve or disrupt brain functions and can therefore be used to investigate hemispheric specialization. Accordingly, this study was designed to research hemispheric specialization in the control of final position accuracy by comparing the effects of tDCS on dorsolateral prefrontal cortex (DLPFC) in the right and left hemispheres. Forty-three right-handed male university students (aged $21.34 \pm 1.61)$ volunteered to participate in this study. They were divided into the right DLPFC, left DLPFC, sham, and practice groups, including 12,11, 9, and 11 participants, respectively. After learning motor skills in two days, the participants practiced final position accuracy in one day. They were asked to move the cursor toward the centers of targets appearing randomly at the top, middle, and bottom on the right side of a monitor as accurately and quickly as they could. At the time of practice, the participants received anodic stimulation in one hemisphere and cathodic stimulation in the other. The results indicated that the left anodic/right cathodic group (left DLPFC) showed the worst performance, which may be caused by the inhibitory effects of cathodic stimulations in the right DLPFC. Therefore, it is predicted that the right hemisphere may have greater specialization in final position accuracy of movement.
\end{abstract}

Keywords: dorsolateral prefrontal cortex, hemispheric specialization, final position accuracy, transcranial direct current stimulation.

\section{Introduction}

It has been indicated that each part of the brain has different functions, which is defined as the specializations of different parts of the brain for different actions. Regarding hemispheric specialization, recent studies propose different functions for each hemisphere of the brain (De Schotten et al., 2011; Duff \& Sainburg, 2007; Haaland, Prestopnik, Knight, \& Lee, 2004; Jason, 1983; Mutha, Haaland, \& Sainburg, 2012; Mutha, Sainburg, \& Haaland, 2011a, 2011b; R. Sainburg \& Kalakanis, 2000; R. L. Sainburg, 2005; R. L. Sainburg, Schaefer, \& Yadav, 2016; Schaefer, Haaland, \& Sainburg, 2007, 2009; Schaefer, Mutha, Haaland, \& Sainburg, 2012; Schambra et al., 2011; Yadav \& Sainburg, 2011). For example, Mutha et al. (2011a) investigated patients with damage to the left or right hemispheres to emphasize left- hemispheric specialization in adaptation to deviations in the initial motion direction and right-hemispheric specialization in disruption correction during movement. Adaptation to deviations in the initial motion direction in the first part of a reaching movement indicates the left-hemispheric specialization in feedforward mechanisms and motor programming by the closed-loop control system. In contrast, correcting disparities created during movement by the right hemisphere shows the right-hemispheric specialization in the use of feedback mechanisms and the closed-loop control system. Some studies of lateralization and motor learning, such as Stöckel and Weigelt (2012), pointed out the laterality of the left hemisphere in controlling feedforward mechanisms and motor programming (such as sequence and timing control and trajectory control) as well as the laterality of the right hemisphere in spatial processes (such as final position accuracy). Generally, the results of some studies conducted on patients with unilateral brain lesions, indicated the left-hemispheric specialization in 
controlling the programming features of the first movement phase by using feedforward mechanisms. They also showed the right-hemispheric specialization in the accuracy of final position in the second movement phase by using a feedback mechanism (Mutha et al., 2012; Schaefer et al., 2009; Schaefer et al., 2012). More importantly, previous studies emphasized the laterality of the right hemisphere in the use of proprioception (Goble \& Brown, 2008) and visual feedback (Stöckel \& Wang, 2011). It appears that the right-hemisphere circuits have more important roles in the accuracy of the final position by adjusting the mechanisms of sensorimotor consolidation (Mutha et al., 2012; Mutha et al., 2011a). This is very important in many of the fast movements requiring accuracy, such as reaching movements.

Reaching movements consist of two steps. The first includes the increase in acceleration relating to preplanned mechanisms and motor programming. It forms the first part of a reaching movement. The second step is the decrease in acceleration depending on feedback mechanisms and sensory feedback (R. L. Sainburg \& Schaefer, 2004). In fact, the initial acceleration is a reflection of open-loop processing, which programs the best sequence of muscular activities. The decreased acceleration is a reflection of closed-loop processing, which uses feedback for online intelligent configurations and increased accuracy of movement to attain the target accurately (Haaland et al., 2004; R. L. Sainburg, 2005). According to Yadav and Sainburg's hybrid theory of motion control (2011), it is predicted that open-loop and motor programming mechanisms have more specializations in the left hemisphere, whereas feedback and final position accuracy mechanisms have more specializations in the right hemisphere (Yadav \& Sainburg, 2011). Our previous study confirmed the assumption of the left-hemispheric specialization in motor programming process in healthy individuals by using tDCS (Teymuri, Saberi, Taheri, Ghanaie, \& Darainy, 2017). However, whether healthy individuals benefit from a particular hemispheric specialization in the accuracy of final position is a question that researchers intend to address by using tDCS in the current study.

Transcranial direct currency stimulation (tDCS) is a non-invasive and painless method that increases neural activity through resting membrane depolarization in the anodic mode. Also, It results in deterioration and decreases neural activity through resting membrane hyperpolarization in the cathodic method (Hunter, Sacco, Nitsche, \& Turner, 2009; Keeser et al., 2011; M. Nitsche \& Paulus, 2000; M. A. Nitsche et al., 2008; M. A. Nitsche, Schauenburg, et al., 2003; Reis \& Fritsch, 2011). The effects of tDCS on motor learning have been confirmed by many studies (Hunter et al., 2009; M. A. Nitsche, Schauenburg, et al., 2003; Reis \& Fritsch, 2011; Schambra et al., 2011). It has been proposed that tDCS can improve and weaken motor learning and performance by making structural and functional changes to the cortical membrane (Utz, Dimova, Oppenländer, \& Kerkhoff, 2010) in the anodic and cathodic conditions (M. Nitsche \& Paulus, 2000; M. A. Nitsche et al., 2008; M. A. Nitsche, Schauenburg et al., 2003; Reis \& Fritsch, 2011). This feature has turned tDCS into a tool that can be used to understand the specializations of different parts of the brain in motor learning and control (Schambra et al., 2011). In our previous study, tDCS was used to determine that the left M1 had more specializations in motor programming than the right M1 did (Teymuri et al., 2017). Schambra et al. (2011) used tDCS to investigate the hemispheric specialization in motor-skills learning and emphasized the left-hemispheric specialization in motor learning. Therefore, the aim of this study is to investigate the hemispheric specialization in the accuracy of target attainment in the final position. The result can be used to help individuals having problems attaining targets accurately from an applied perspective. It also helps understanding of how hemispheric specialization can be useful in motor learning from a theoretical perspective.

\section{Materials and Methods}

Forty-three male university students (aged $20.44 \pm 1.57$ years old) volunteered to fill out the informed consent to participate in this study. The study approved by our Faculty of Physical Education and Sport Sciences (code: 3/41014) and all experiments were performed in the Motor Behavior Laboratory of our Faculty. According to the inclusion criteria, the participants should be right-handed, not have any upper-extremity musculoskeletal lesions, have adequate power of sight, and not take any psychotropic drugs. The participants were divided into four groups at random. In the right DLPFC groups $(\mathrm{n}=12)$, the participants practiced motor skills at the time of right-hemispheric stimulation. In the left DLPFC $(n=11)$, the participants practiced motor skills at the time of left-hemispheric stimulation. In the sham group $(n=9)$, the participants practiced motor skills without receiving any stimulations while electrodes were attached to their heads. In the practice group $(n=11)$, the participants practiced motor skills without any stimulation in the right or left hemispheres. 


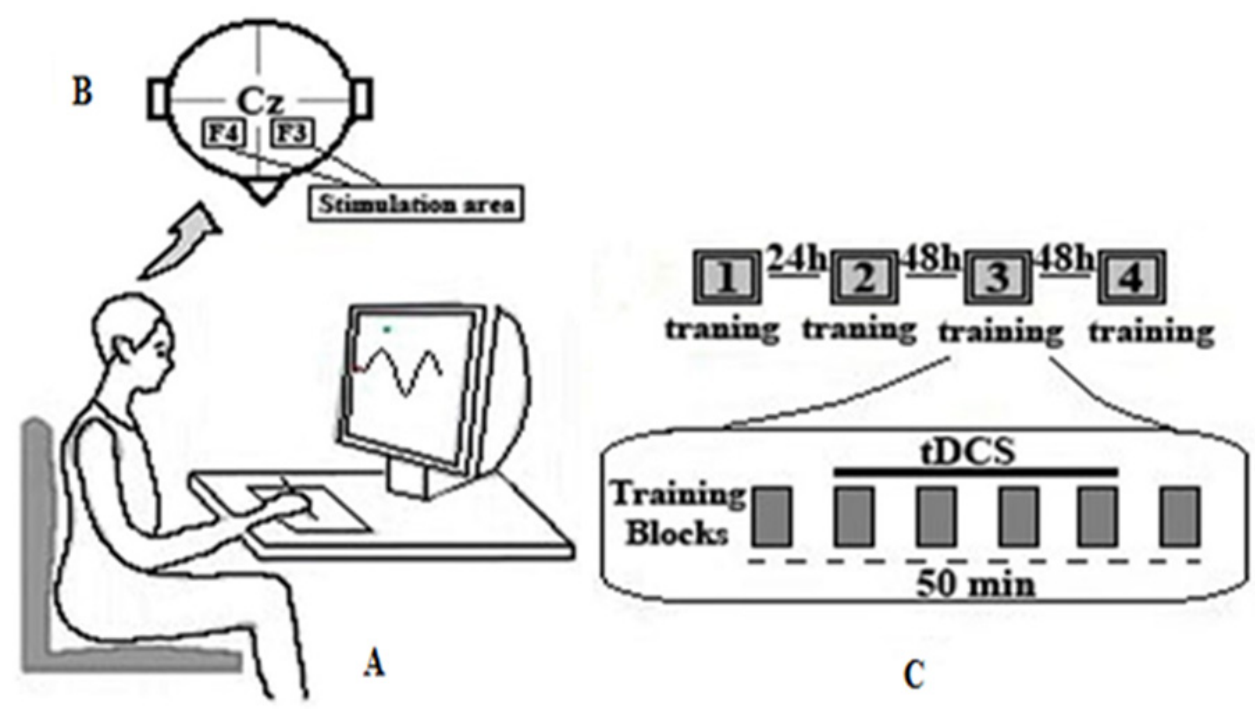

Figure 1. Method

A - General Research Schema: The four groups used a light pen to do the task on a monitor ( $9^{*} 12$ inches). B Stimulation Paradigm: During the practice, the anode was placed on F3, and the cathode was placed on F4 for the left DLPFC stimulation. They were swapped for the right DLPFC stimulation. C- Practice Paradigm: After learning the paradigms, the participants participated in a pretest and a practice session. The acquisition and retention tests were conducted after 10 minutes and 48 hours, respectively.

\subsection{Task}

The participants were asked to sit at a computer desk to use a light pen (Genius Pen Sketch M912A, 9*12 inches, 5120 LPI, 2048 pressure sensitivity) to redraw the motor paradigms (taken from Wulf and Schmidt, 1994) appearing randomly (Figure 1a). For this purpose, the participants were required to put the cursor on the start by using the light pen and then draw the observed paradigm. The end of the paradigm was specified with an invisible line. The participants were allowed to see the paradigm for one second, and then it disappeared. After that, they were asked to draw it quickly and accurately. Moreover, the cursor of the light pen did not leave any traces on the screen at the time of drawing the paradigm. This approach created conditions that made individuals draw the paradigms by using the feedforward information and motor programming. Then the criterion paradigm was displayed for individuals along with the drawn paradigm so that they could make corrections in the next move. After practicing the paradigms for two days, the participants were asked to accurately and quickly move the cursor towards targets as thick as $1.5 \mathrm{~cm}$ appearing in three points (top, middle, and bottom) on the right side of the screen (Figure 2). At the end of the movement, the final accessibility point of a participant was shown with a small red point, the distance of which from the target was regarded as the static error. It should be noted that these targets appear after the movement starts and when an individual enters the final one-third segment of the paradigm. This prevents participants from programming the implementation of the paradigm and attaining the target at the same time in one motor program by using feedforward information. Instead, participants were encouraged to use visual information and move the hand towards the target online. The data pertaining to static error, movement time, and the ratio of error to time and skill (Equation 1) were measured as the following criteria for evaluation.

$$
\text { Skill }=1 / \text { Error* } 1 / \text { Movement time } * 100
$$




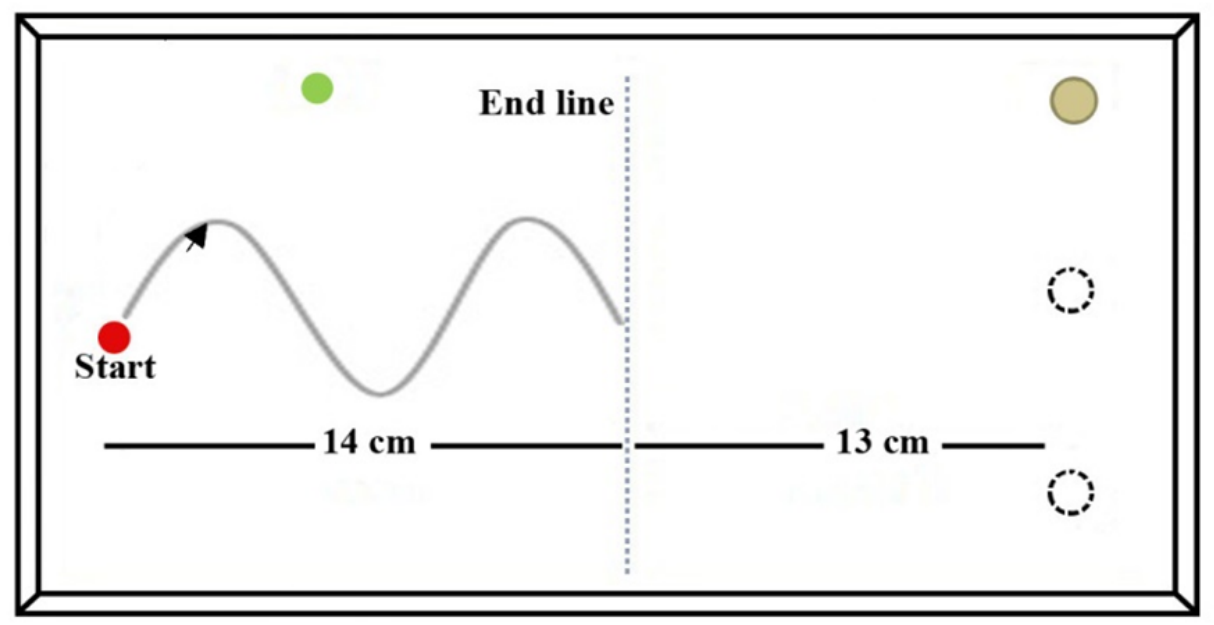

Figure 2. Motor Task: The paradigms are presented to participants at random so that they start drawing After learning the paradigms in accordance with the protocol of our previous study (Teymuri et al. 2017), the participants practiced reaching the hand to the final position accuracy. The targets appeared at the top, middle, and bottom right side of the screen at random. The participants were asked to reach their hands to them accurately and quickly. The distance between the final accessibility point and target was regarded as the static error, and the distance between the invisible end line and the accessibility point was regarded as the movement time.

\subsection{Stimulation}

Since the frontal areas (Mutha et al., 2011a; Shadmehr \& Holcomb, 1997) and especially the dorsolateral prefrontal cortex (DPFC) (Shadmehr \& Holcomb, 1997) are more important in motion accuracy, the current study used this area to investigate the hemispheric specialization in final position accuracy. Two $25-\mathrm{cm}^{2}$ electrodes, soaked in a saline solution, were used for simulation with tDCS (oasis pro; mind alive device). Since bihemispheric tDCS (anodic and cathodic) results in more behavioral effects than the unihemispheric stimulation (Lindenberg, Renga, Zhu, Nair, \& Schlaug, 2010; Vines, Cerruti, \& Schlaug, 2008), the 10-20 system was used for the left anodic DLPFC on F3 and cathodic DLPFC on F4 as well as the right anodic DLPFC on F4 and cathodic DLPFC on F3 (Figure 1b). Then tDCS with $2 \mathrm{~mA}$ voltage was used for 20 minutes with the currency density of 0.08 $\mathrm{mA} / \mathrm{cm}^{2}$ for each electrode during the practice. In the sham group, the practice lasted for 15 seconds (Gandiga, Hummel, \& Cohen, 2006; Schambra et al., 2011). During every practice session, the participants were repeatedly asked about the possible side effects (such as headaches, skin problems, inattention, and drowsiness) of the stimulation.

\subsection{Practice Paradigm}

After learning the patterns (in accordance with the practice paradigm in the previous study, reference), the participants took part in a five-trial pretest and then a session including six 30-trial blocks with 30 -second breaks between the two blocks. They participated in an acquisition test after 10 minutes and in a retention test after 48 hours (Figure 1c). In this study, the participants were asked to focus on the learning accuracy of target attainment and like the study conducted by Schambra et al. (2011), they received 20 minutes of stimulation with practice along four middle blocks.

\subsection{Psychophysical Assessment}

The information pertaining to the participants' sleep time (Schambra et al., 2011), visualization ability (C. R. Hall \& Martin, 1997), and handedness (Oldfield, 1971) can be seen in Table 1. They were also asked to describe the feelings caused by the use of tDCS physically, mentally, and behaviorally on a sheet of perception.

\subsection{Statistical Analysis}

Since the design of research includes four groups in three time periods, ANOVA was used with repeated measures to compare static error, movement time, the ratio of static error to movement time, and the skills of four groups (left DLPFC, right DLPFC, sham tDCS, and practice) in three time periods (pretest, acquisition, and retention). The Bonferroni test and one-way ANOVA were used for pairwise comparisons and group comparisons, respectively. Moreover, the one-way ANOVA was conducted to compare demographics between groups. The 
significance was evaluated at $\mathrm{p} \leq 0.05$ in two tails.

\section{Results}

The demographics of individuals, age, visualization ability, handedness, and sleep time can be seen in Table 1 . The one-way ANOVA test did not show any significant differences between groups in any variables $(p>0.05)$.

Table 1. Demographic and Psychological Characteristics of Participants

\begin{tabular}{llllll}
\hline Group & $\mathbf{N}^{\mathbf{a}}$ & Age(year) $^{\mathbf{b}}$ & Imagery ability $^{\mathbf{c}}$ & Handedness $^{\mathbf{d}}$ & Sleep duration(h) $^{\mathbf{e}}$ \\
\hline Right DLPFC & 12 & $20.41 \pm 1.50$ & $46.83 \pm 7.27$ & $93.33 \pm 6.51$ & $7.01 \pm 1.20$ \\
Left DLPFC & 11 & $20.09 \pm 1.30$ & $47.36 \pm 6.98$ & $94.54 \pm 6.87$ & $7.36 \pm 1.02$ \\
Sham $t$ DCS & 9 & $20.22 \pm 2.16$ & $46.55 \pm 6.52$ & $97.77 \pm 4.40$ & $7.00 \pm 0.86$ \\
Practice & 11 & $21.10 \pm 1.41$ & $44.81 \pm 6.17$ & $95.45 \pm 6.78$ & $7.45 \pm 0.93$ \\
v value $^{g}$ & & 0.56 & 0.63 & 0.83 & 0.45 \\
\hline
\end{tabular}

a: the number of individuals in each group; b: age in year; c: the visualization ability of movement was evaluated by using Hall and Martin's Visualization Ability Questionnaire (1997) including 8 items, each of which had 1 score for the lowest ability and 7 for the highest ability; $\mathbf{d}$ : handedness was measured by using the Edinburg test having 10 items ranging from 0 to 100 ; e: sleep time in hour; and g: difference in the means of groups was measured in each variable by using the one-way ANOVA.

First, the one-way ANOVA was used to evaluate RMSE in four groups in the retention test. The results showed that the groups were not significantly different in the retention test ( $>0.05)$, and they learned from paradigms in the same way. Then the data of static error, the ratio of static error to movement time and skill were analyzed by using repeated measure ANOVA.

\subsection{Static Error}

The static errors of the four groups (left DLPFC, right DLPFC, tDCS sham, practice) were analyzed by using ANOVA with repeated measure at three times (pretest, acquisition, and retention). The results indicated that the effect of time $\left(f_{(2,78)}=42.398, P=0.000, \eta^{2}=0.521\right)$ and the effect of group $\left(f_{(3,39)}=10.185, P=0.000, \eta^{2}=0.439\right)$ were significant, so that the left DLPFC group was significantly different from all the other groups $(\mathrm{p} \leq 0.05)$. Moreover, the interaction of time* group was significant $\left(\mathrm{f}_{(6,78)}=2.761, \mathrm{P}=0.017, \eta^{2}=0.175\right)$. After that, the groups were investigated in each stage by using the one-way ANOVA. Although the groups were not significantly different in the pretest $\left(\mathrm{f}_{(3)}=0.159, \mathrm{P}=0.923\right)$, they were significantly different in the acquisition $\left(\mathrm{f}_{(3)}=33.262, \mathrm{P}=\right.$ 0.000 ) and retention ( $\left.\mathrm{f}_{(3)}=3.434, \mathrm{P}=0.026\right)$ tests. The Bonferroni post hoc test showed this difference in the acquisition between the left DLPFC and other groups $(\mathrm{p} \leq 0.05)$. It also showed a difference between the right DLPFC and left DLPFC in the retention test. As indicated in Figure 3a, the left DLPFC showed the worst performance in the acquisition and retention tests, which indicates the lack of improvement in the static error in this group.

\subsection{Movement Time}

The data of the movement times of the four groups in three times were analyzed with repeated measure ANOVA. The results indicated that the effects of time $\left(f_{(2,78)}=6.169, P=0.003, \eta^{2}=0.137\right)$ was significant. However, the effects of groups $\left(\mathrm{f}_{(3,39)}=0.863, \mathrm{P}=0.469, \eta^{2}=0.062\right)$ and the interaction of time* $\operatorname{group}\left(\mathrm{f}_{(6,78)}=0.485, \mathrm{P}=0.817\right.$, $\left.\eta^{2}=0.036\right)$ were not significant. Then the groups were investigated by using the one-way ANOVA in each stage. The results showed that they were not significantly different in any of the stages $(\mathrm{p}>0.05)$.

\subsection{Error/Time}

The results showed that the effect of time $\left(\mathrm{f}_{(2,78)}=46.608, \mathrm{P}=0.000, \eta^{2}=0.544\right)$ was significant, insofar as significant differences were observed in all stages $(\mathrm{p} \leq 0.05)$. Moreover, the effect of group was significant $\left(\mathrm{f}_{(3,39)}=\right.$ $\left.12.092, \mathrm{P}=0.000, \eta^{2}=0.482\right)$, insofar as the left DLPFC group was significantly different from other groups $(\mathrm{p} \leq 0.05)$. Moreover, the interaction of time* group was significant $\left(\mathrm{f}_{(6,78)}=3.046, \mathrm{P}=0.010, \eta^{2}=0.190\right)$. After that, the groups were investigated by using the one-way ANOVA in each stage. Although the groups were not significantly different in the pretest $\left(\mathrm{f}_{(3)}=1.511, \mathrm{P}=0.227\right)$, they were significantly different in the acquisition $\left(\mathrm{f}_{(3)}\right.$ $=24.155, \mathrm{P}=0.000)$ and retention stages $(\mathrm{f}(3)=5.340, \mathrm{P}=0.004)$. The Bonferroni post hoc test showed this difference in the acquisition stage between the left DLPFC and other groups $(\mathrm{p} \leq 0.05)$. It also showed this difference between the left DLPFC and right DLPFC as well as the left DLPFC and the sham group in the retention 
stage. According to Figure 3b, the ratio of error to movement time was higher in the left DLPFC in the acquisition and retention stages.

\subsection{Skill}

Skill was defined as the quick and accurate implementation of the task. The results indicated that the effect of time $\left(f_{(2,78)}=92.758, P=0.000, \eta^{2}=0.704\right)$ was significant, insofar as significant differences were observed between all stages $(p \leq 0.05)$. The effect of group $\left(f_{(3,39)}=14.077, P=0.000, \eta^{2}=0.520\right)$ was significant, too, something regarded as a difference between the left DLPFC and other groups by the Bonferroni post hoc test $\left(\mathrm{f}_{(6,78)}=10.119, \mathrm{P}=0.000\right.$, $\eta^{2}=0.438$ ). After that, the groups were investigated by using the one-way ANOVA. Although the groups were not significantly different in the pretest $\left(\mathrm{f}_{(3)}=0.118, \mathrm{P}=0.949\right)$ they were only significantly different in the acquisition stage $\left(\mathrm{f}_{(3)}=13.898, \mathrm{P}=0.000\right)$. The Bonferroni post hoc test indicated this difference between the left DLPFC and other groups $(\mathrm{p} \leq 0.05)$. According to Figure $3 \mathrm{c}$, the left DLPFC show lower levels of skill compared with other groups.

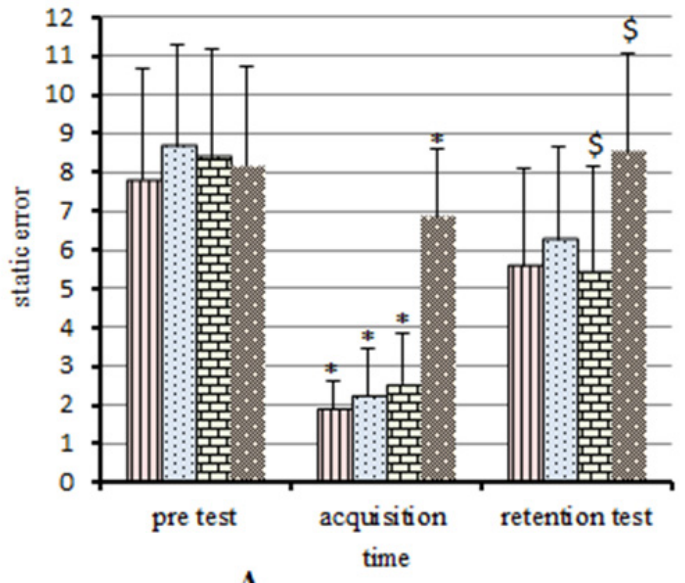

A

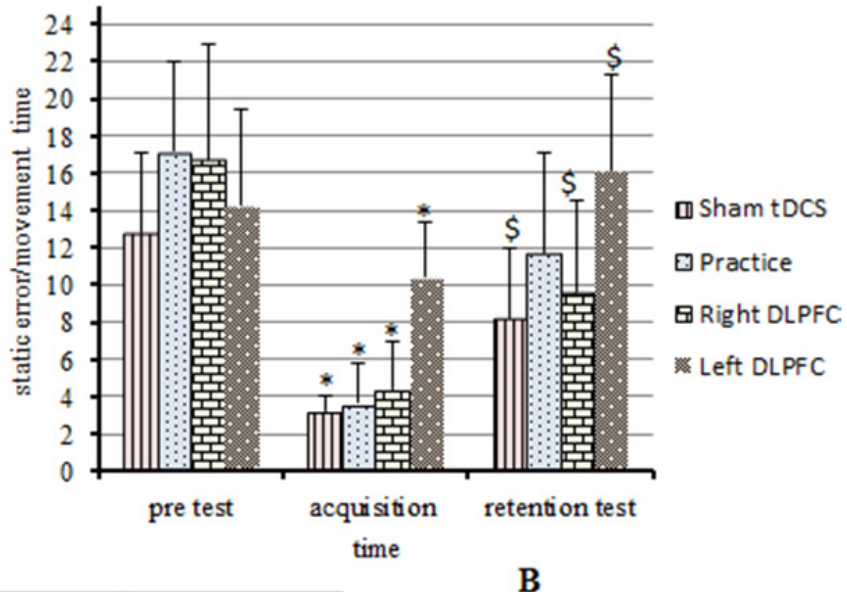

B

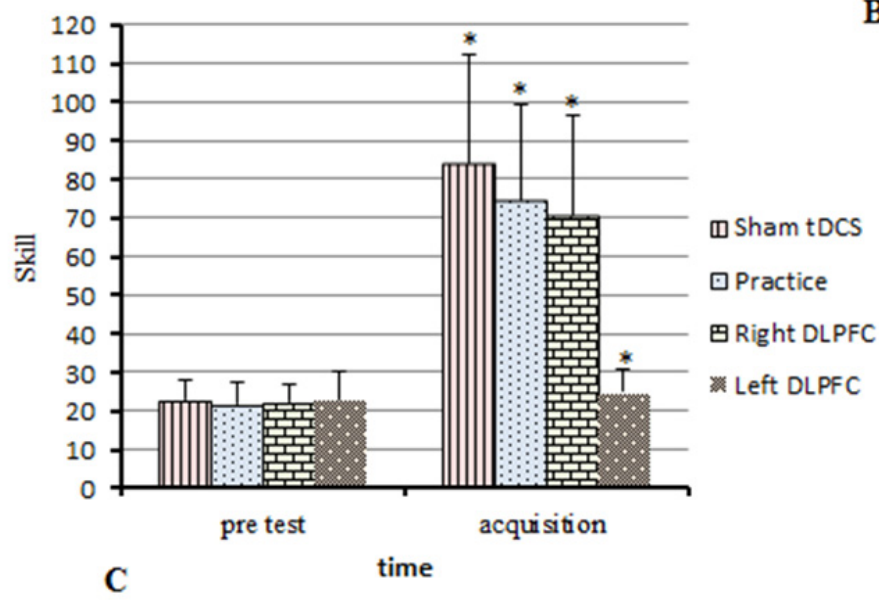

Figure 3.

A: the scores of static errors of the four groups can be seen in the pretest, acquisition, and retention tests. $*$ The Left DPLFC is significantly different from other groups in the acquisition stage. \$ The Left DLPFC is significantly different from the Right DLPFC in the retention stage, insofar as the Left DLPFC showed higher errors in both stages. B: the ratio of static error to the movement time pertaining to the four groups can be seen in the pretest, acquisition, and retention stages. *In the acquisition stage, the Left DLPFC group is significantly different from other groups. \$ The Left DLPFC was significantly different from the Right DLPFC and the sham group in the retention stage, insofar as the Left DLPFC showed higher ratios in both stages. C: The skill scores can be seen in the pretest and acquisition stages in the four groups. * In the acquisition stage, the Left DLPFC was significantly different from other groups in the acquisition stage, insofar as it showed lower levels of skills than other groups. 


\section{Discussion}

The results of this study showed that the left anodic/right cathodic stimulation on DLPFC resulted in more static errors in the acquisition and retention stages compared with the other state. This can be caused by the deteriorative effects of the right cathodic stimulation. Regarding the movement time, the groups were not significantly different from each other, meaning that the movement error did not increase by increasing the movement time (i.e. a speed-accuracy trade-off). Regarding the most important variable, i.e. skill including accuracy and speed, the cathodic left-hemispheric stimulation decreased this variable significantly in the acquisition stage. However, it did not create any significant differences between the groups in the retention stage. These results support the right-hemispheric specialization in the control of final position accuracy.

\subsection{The Right-Hemispheric Specialization in the Final Position Accuracy}

Regarding the laterality of different parts of brain, the results of previous studies propose different functions, which are defined as the specialization of different parts of the nervous system for different actions (De Schotten et al., 2011; Duff \& Sainburg, 2007; Haaland et al., 2004; Mutha et al., 2012; Mutha et al., 2011a, 2011b; R. Sainburg \& Kalakanis, 2000; R. L. Sainburg, 2005; R. L. Sainburg et al., 2016; Schaefer et al., 2007, 2009; Schaefer et al., 2012; Schambra et al., 2011; Yadav \& Sainburg, 2011). Reviewing previous studies conducted on the specialization of brain showed interesting results regarding the role of each hemisphere in the control of different aspects of skill. Mutha et al. ( 2011a) showed that damage to the right hemispheric frontal cortex could result in the inability to correct discrepancies during a movement. In fact, the results of their study pointed out more specialization of the right-hemispheric frontal cortex in the use of feedback information and the correction of discrepancies during the movement. In favor of this issue, the results of many studies (Mutha et al., 2011b; Schaefer et al., 2009; Schaefer et al., 2012) conducted on the patients with unilateral brain lesions indicated that the right hemisphere is mainly responsible for the control mechanisms during execution phase, but not feedforward mechanisms before the implementation. Therefore, damage to the right hemisphere can result in the inability to finish a movement accurately. Stöckel and Wang (2011) created laboratory conditions in their study to provide individuals with some information to force them to focus on the visual-motor features of a task. In such conditions, the left-to-right transfer was greater than the right-to-left transfer. Since each hemisphere controls the organs on the opposite side of the body (J. E. Hall, 2015), the laterality of transferring spatial information from left to right can show more specialization of the left hemisphere/right foot in the control of spatial information and the accuracy of movement. Stöckel and Weigelt (2012) investigated the hemispheric laterality and motor learning, too, and emphasized the greater right-hemispheric specialization in spatial processing. More importantly, the right hemisphere uses the feedback information on proprioception (Goble \& Brown, 2008) and visual (Stöckel \& Wang, 2011) to a greater extent. Moreover, the right-hemisphere circuits have greater roles in the accuracy of the final position by adjusting the sensorimotor fixation mechanisms (Mutha et al., 2012; Mutha et al., 2011a). Overall, this evidence can indicate the greater right-hemispheric specialization in processing sensory information and feedback mechanisms for the improvement in the final position accuracy. All of these studies were conducted on patients with unilateral brain lesions. However, it is still unknown how the hemispheric specialization operates in the accuracy of final position in healthy individuals. Accordingly, the current study like a study conducted by Schambra et al. (2011) used tDCS to investigate the hemispheric specialization. The results are consistent with the abovementioned findings emphasizing the right-hemispheric specialization in the final position accuracy.

\subsection{The Effects of tDCS and Right-Hemispheric Specialization in Final Position Accuracy}

The anodic stimulation can increase excitability and automatic neural firing. This process is caused by depolarization in the potential of resting membrane and by facilitating the function of the stimulated area. On the other hand, the cathodic stimulation can decrease excitability and neural firing, a process caused by hyperpolarization in the potential of resting membrane and by the deterioration in the function of the stimulated area (M. Nitsche \& Paulus, 2000; M. A. Nitsche et al., 2008; M. A. Nitsche, Nitsche, et al., 2003). In fact, as anodic stimulation can increase the excitability of motor membrane by increasing the stimulus neurotransmitters or decreasing the inhibitory neurotransmitters, the cathodic stimulation can decrease the excitability of motor membrane by decreasing the stimulus neurotransmitters or increasing the inhibitory neurotransmitters (Reis \& Fritsch, 2011; Reis et al., 2008; Stagg et al., 2009). For example, Stagg et al. (2009) investigated the changes in the concentration of neurotransmitters as a result of tDCS. They reported that the anodic stimulation decreased GABA; however, the cathodic stimulation decreased glutamate. In addition, the cathodic stimulation can change the function of the nervous membrane. Apart from local changes in ionic concentrations, these changes can be caused by the changes pertaining to membrane-transferring proteins and the changes pertaining to electrolytes such as $\left[\mathrm{H}^{+}\right]$ (Ardolino, Bossi, Barbieri, \& Priori, 2005). Since the effects of tDCS are not significant per se(Antal et al., 2004; 
Galea \& Celnik, 2009), it is advised to apply them by practice so that they have greater and longer effects (Reis \& Fritsch, 2011).

At the time of acquiring motor skills, the effects of tDCS can be different with respect to conditions (Fregni et al., 2005), cathodic or anodic stimulation (Vines, Nair, \& Schlaug, 2008), and the type of task (Mansouri et al., 2016). For example, Fregni et al. (2005) conducted a study on stroke patients to show that increased activities in a damaged hemisphere could improve recovery; however, extra activities in a healthy hemisphere might lead to an inconsistent strategy. Saucedo Marquez, Zhang, Swinnen, Meesen, and Wenderoth (2013) confirmed different effects of tDCS on the sequential finger tapping task (SEQTAP) and the visual isometric pinch force task (FORCE) Mansouri et al. (2016) indicated that the effects of tDCS on DLPFC could be different with respect to the type of task. In the Wisconsin Card Sorting Test (WCST), the rules of matching items continuously change, and individuals discover these changes by trial and error. In the stop task, participants are supposed to respond to a go-sign quickly; however, they should stop responding when the stop-sign appears. The results indicated that the cathodic and anodic stimulations of DLPFC could result in the greatest effects on WCST and stop tasks, respectively. In WCST and stop tasks, the adjustments reflect different processes involved in the adjustment of transferring rules pertaining to behavior and response-inhibition control, respectively. A pole of tDCS, which can have the greatest effect, also differs between the two tasks. Therefore, the adjustments of tDCS depend on the polarity and features of the tasks (Mansouri et al., 2016). The results of previous studies indicated that the anodic stimulation of left M1 (left anodic/right cathodic) resulted in more skills of motor program in the retention test. However, the current study showed that the cathodic stimulation of right DLPFC (left anodic/right cathodic) prevented improvement in the skill of final position accuracy in the acquisition test. Therefore, the previous study emphasized left-hemispheric specialization in motor programming, and the current study emphasizes righthemispheric specialization in the accuracy of final position. However, it is predicted that the controlling mechanisms of each of the two motor programming and final position accuracy can probably be different. Perhaps, the motor programming process depends on the motor programs learned by the motor memory (nervous traces), and anodic stimulation can strengthen this memory and nervous traces; therefore, it can improve the motor programming process. To confirm this, the effects of tDCS on the improvement in coding processes in motor memory in the primary motor cortex (Bütefisch, Khurana, Kopylev, \& Cohen, 2004) and the fixation of this motor memory have been emphasized (Reisa et al., 2009). On the contrary, it is predicted that the target attainment process at the final position is done by online and feedback mechanisms that are mostly influenced by the inhibitory mechanisms of cathodic stimulation. The abovementioned interpretations are consistent with the results of Cantarero et al. (2015) who reported that anodic stimulation had more effects on consolidation and learning (retention) the skill, and cathodic stimulation had more effects on online processes (acquisition). However, more investigation is required to understand and prove how these mechanisms work.

\section{General Discussion}

The results of our previous study (Teymuri et al. 2017) indicated that the left hemisphere had laterality in the motor programming process by using feedforward information; however, the results of the current study support the right-hemispheric specialization in the final position accuracy by using online feedback information.

The results of different studies indicate that performing the movements that require speed and accuracy depends on the control of two open-loop and closed-loop systems (Serrien, Ivry, \& Swinnen, 2006). For example, the first part of a reaching movement (i.e. increasing acceleration) is controlled by feedforward processes and motor programming before performing the movement through the open-loop control system. However, the final part of a movement and target attainment (i.e. decreasing acceleration) is controlled by feedback processes and closed-loop control (Haaland et al., 2004; R. L. Sainburg, 2005; R. L. Sainburg \& Schaefer, 2004). In the dynamic dominance theory (R. L. Sainburg, 2002), Sainburg believes that the open-loop control mechanisms are probably processed more in the left hemisphere and the closed-loop control mechanisms are probably processed more in the right hemisphere. In the previous study (Teymuri et al. 2017), the motor paradigms were used along with special conditions that individuals induced to program the paradigm and movement trajectory with the help of feedforward information and predictive mechanisms. Accordingly, the results indicated that the left hemisphere had greater specialization in motor programming. On the contrary, some conditions were provided in the current study to encourage people to use online feedback information to attain the targets appearing on the right side of the monitor quickly and accurately. Therefore, the results indicated that the right hemisphere had greater specialization in final position accuracy. These results are consistent with the current hypotheses regarding the hemispheric specialization of the brain. The hypothesis of hybrid specialization, introduced by Yadav and Sainburg (2011), includes two predictive control and impedance control. The predictive control states that the left hemisphere has a more prominent role in planning and controlling movements by using feedforward information. While, the 
impedance control points out that the right hemisphere has greater specialization in the correction of errors and accurate target attainment at the end of movement. This finding is also consistent with the model introduced by MacNeilage, Rogers, and Vallortigara (2009). This model was based on the evidence obtained from a wide range of motor behaviors pertaining to many types of vertebrates. In this model, it is proposed that the left hemisphere has greater specialization for the well-established paradigms of the behaviors performed in familiar conditions, and the right hemisphere has greater specialization to respond to unpredictable environmental events.

\section{Conclusion}

Overall, it appears that the left hemisphere has greater specialization in planning and controlling the first part of a reaching movement, while the right hemisphere has greater specialization in the final position accuracy in the second part of reaching movement. In addition, it is predicted that they have different controlling mechanisms because the anodic stimulation of the left hemisphere can improve motor programming more. However, the cathodic stimulation of the right hemisphere can result in learning deterioration in target attainment at the final position of movement. Proving the fact that each of the two parts of a movement is controlled by two different hemispheres of the brain can be very important in both applied and theoretical ways. From an applied standpoint, it may help the patients having problems in each of these two parts by stimulating or deteriorating the involved areas such as in patients with Down syndrome who appear to have problems in the programming part. From a theoretical standpoint, it can lead to a research area that improves our perception of how movement is controlled by the central nervous system.

\section{Future Investigations}

Fist, since the participants in this study and other similar studies were all right-handed individuals, the question is how much this specialization is influenced by the right hand. Therefore, it is necessary to investigate the left-hemispheric specialization in the control of the initial part and the right hemisphere in the final position accuracy in the left-handed individuals. Second, the anodic stimulation of the left hemisphere, compared with the right hemisphere and the control group, improved motor programming in the previous study. Moreover, the cathodic stimulation of the right hemisphere, compared with the anodic stimulation of the left hemisphere and the control group, resulted in the lack of learning final position accuracy. Therefore, the assumption of the left-hemispheric specialization in motor planning and the right hemispheric in the final position accuracy has been confirmed. However, more investigation should be conducted to determine the mechanisms involved in each of these processes. Third, it appears that the movements are stored in the parietal areas of the left hemisphere as they are learned (Halsband \& Lange, 2006; Mutha et al., 2011a). In fact, the right hemisphere has specialization by using visual feedback and correcting the errors in updating and consolidation motor skills in the left hemisphere, something that requires more investigation.

\section{Competing Interests Statement}

The authors declare that they has no competing or potential conflicts of interest.

\section{References}

Antal, A., Nitsche, M. A., Kincses, T. Z., Kruse, W., Hoffmann, K. P., \& Paulus, W. (2004). Facilitation of visuomotor learning by transcranial direct current stimulation of the motor and extrastriate visual areas in humans. European Journal of Neuroscience, 19(10), 2888-2892. https://doi.org/10.1111/j.1460-9568.2004.03367.x

Ardolino, G., Bossi, B., Barbieri, S., \& Priori, A. (2005). Non - synaptic mechanisms underlie the after-effects of cathodal transcutaneous direct current stimulation of the human brain. The Journal of physiology, 568(2), 653-663. https://doi.org/10.1113/jphysiol.2005.088310

Bütefisch, C. M., Khurana, V., Kopylev, L., \& Cohen, L. G. (2004). Enhancing encoding of a motor memory in the primary motor cortex by cortical stimulation. Journal of Neurophysiology, 91(5), 2110-2116. https://doi.org/10.1152/jn.01038.2003

Cantarero, G., Spampinato, D., Reis, J., Ajagbe, L., Thompson, T., Kulkarni, K., \& Celnik, P. (2015). Cerebellar direct current stimulation enhances on-line motor skill acquisition through an effect on accuracy. The Journal of Neuroscience, 35(7), 3285-3290. https://doi.org/10.1523/JNEUROSCI.2885-14.2015

De Schotten, M. T., Dell'Acqua, F., Forkel, S. J., Simmons, A., Vergani, F., Murphy, D. G., \& Catani, M. (2011). A lateralized brain network for visuospatial attention. Nature neuroscience, 14(10), 1245-1246. https://doi.org/10.1038/nn.2905

Duff, S. V., \& Sainburg, R. L. (2007). Lateralization of motor adaptation reveals independence in control of trajectory and steady-state position. Experimental brain research, 179(4), 551-561. 
https://doi.org/10.1007/s00221-006-0811-1

Fregni, F., Boggio, P. S., Mansur, C. G., Wagner, T., Ferreira, M. J., Lima, M. C., \& Nitsche, M. A. (2005). Transcranial direct current stimulation of the unaffected hemisphere in stroke patients. Neuroreport, 16(14), 1551-1555. https://doi.org/10.1097/01.wnr.0000177010.44602.5e

Galea, J. M., \& Celnik, P. (2009). Brain polarization enhances the formation and retention of motor memories. Journal of Neurophysiology, 102(1), 294-301. https://doi.org/10.1152/jn.00184.2009

Gandiga, P. C., Hummel, F. C., \& Cohen, L. G. (2006). Transcranial DC stimulation (tDCS): a tool for double-blind sham-controlled clinical studies in brain stimulation. Clinical Neurophysiology, 117(4), 845-850. https://doi.org/10.1016/j.clinph.2005.12.003

Goble, D. J., \& Brown, S. H. (2008). Upper limb asymmetries in the matching of proprioceptive versus visual targets. Journal of Neurophysiology, 99(6), 3063-3074. https://doi.org/10.1152/jn.90259.2008

Haaland, K. Y., Prestopnik, J. L., Knight, R. T., \& Lee, R. R. (2004). Hemispheric asymmetries for kinematic and positional aspects of reaching. Brain, 127(5), 1145-1158. https://doi.org/ 10.1093/brain/awh133

Hall, C. R., \& Martin, K. A. (1997). Measuring movement imagery abilities: A revision of the Movement Imagery Questionnaire. Journal of mental imagery, Vol 21(1-2), 1997, 143-154.

Hall, J. E. (2015). Guyton and Hall textbook of medical physiology: Elsevier Health Sciences, Philadelphia, PA: ISBN: 978-4557-7016-8

Halsband, U., \& Lange, R. K. (2006). Motor learning in man: a review of functional and clinical studies. Journal of Physiology-Paris, 99(4), 414-424. https://doi.org/10.1016/j.jphysparis.2006.03.007

Hunter, T., Sacco, P., Nitsche, M. A., \& Turner, D. L. (2009). Modulation of internal model formation during force field - induced motor learning by anodal transcranial direct current stimulation of primary motor cortex. The Journal of physiology, 587(12), 2949-2961. https://doi.org/10.1113/jphysiol.2009.169284

Jason, G. W. (1983). Hemispheric asymmetries in motor function: I. Left-hemisphere specialization for memory but not performance. Neuropsychologia, 21(1), 35-45. https://doi.org/10.1016/0028-3932(83)90098-2

Keeser, D., Padberg, F., Reisinger, E., Pogarell, O., Kirsch, V., Palm, U., \& Mulert, C. (2011). Prefrontal direct current stimulation modulates resting EEG and event-related potentials in healthy subjects: a standardized low resolution tomography (sLORETA) study. Neuroimage, 55(2), 644-657. https://doi.org/10.1016/j.neuroimage.2010.12.004

Lindenberg, R., Renga, V., Zhu, L., Nair, D., \& Schlaug, G. (2010). Bihemispheric brain stimulation facilitates motor recovery in chronic stroke patients. Neurology, 75(24), 2176-2184. https://doi.org/10.1212/WNL.0b013e318202013a

MacNeilage, P. F., Rogers, L. J., \& Vallortigara, G. (2009). Origins of the left \& right brain. Scientific American, 301(1), 60-67. https://doi.org/10.1038/scientificamerican0709-60

Mansouri, F. A., Fehring, D. J., Feizpour, A., Gaillard, A., Rosa, M., Rajan, R., \& Jaberzadeh, S. (2016). Direct current stimulation of prefrontal cortex modulates error - induced behavioral adjustments. European Journal of Neuroscience. https://doi.org/10.1111/ejn.13281

Mutha, P. K., Haaland, K. Y., \& Sainburg, R. L. (2012). The effects of brain lateralization on motor control and adaptation. Journal of motor behavior, 44(6), 455-469. https://doi.org/10.1080/00222895.2012.747482

Mutha, P. K., Sainburg, R. L., \& Haaland, K. Y. (2011a). Critical neural substrates for correcting unexpected trajectory errors and learning from them. Brain, 134(12), 3647-3661. https://doi.org/10.1093/brain/awr275

Mutha, P. K., Sainburg, R. L., \& Haaland, K. Y. (2011b). Left parietal regions are critical for adaptive visuomotor $\begin{array}{lllll}\text { control. The Journal of } & \text { Neuroscience, }\end{array}$ https://doi.org/10.1523/JNEUROSCI.6432-10.2011

Nitsche, M., \& Paulus, W. (2000). Excitability changes induced in the human motor cortex by weak transcranial direct current stimulation. The Journal of physiology, 527(3), 633-639. https://doi.org/10.1111/j.1469-7793.2000.t01-1-00633.x

Nitsche, M. A., Cohen, L. G., Wassermann, E. M., Priori, A., Lang, N., Antal, A., . . F Fregni, F. (2008). Transcranial direct current stimulation: state of the art 2008. Brain stimulation, 1(3), 206-223. https://doi.org/10.1016/j.brs.2008.06.004 
Nitsche, M. A., Nitsche, M. S., Klein, C. C., Tergau, F., Rothwell, J. C., \& Paulus, W. (2003). Level of action of cathodal DC polarisation induced inhibition of the human motor cortex. Clinical Neurophysiology, 114(4), 600-604. https://doi.org/10.1016/S1388-2457(02)00412-1

Nitsche, M. A., Schauenburg, A., Lang, N., Liebetanz, D., Exner, C., Paulus, W., \& Tergau, F. (2003). Facilitation of implicit motor learning by weak transcranial direct current stimulation of the primary motor cortex in the human. Journal of cognitive neuroscience, 15(4), 619-626. https://doi.org/10.1162/089892903321662994

Oldfield, R. C. (1971). The assessment and analysis of handedness: the Edinburgh inventory. Neuropsychologia, 9(1), 97-113. https://doi.org/10.1016/0028-3932(71)90067-4

Reis, J., \& Fritsch, B. (2011). Modulation of motor performance and motor learning by transcranial direct current stimulation. Current opinion in neurology, 24(6), 590-596. https://doi.org/10.1097/WCO.0b013e32834c3db0

Reis, J., Robertson, E. M., Krakauer, J. W., Rothwell, J., Marshall, L., Gerloff, C., . . Celnik, P. A. (2008). Consensus: Can transcranial direct current stimulation and transcranial magnetic stimulation enhance motor learning and memory formation? Brain stimulation, 1(4), 363-369. https://doi.org/10.1016/j.brs.2008.08.001

Reisa, J., Schambraa, H. M., Cohena, L. G., Bucha, E. R., Fritscha, B., Zarahnc, E., . . Krakauerc, J. W. (2009). Noninvasive cortical stimulation enhances motor skill acquisition over multiple days through an effect on consolidation. PNAS, 106(5). https://doi.org/ 10.1073/pnas.0805413106

Sainburg, R., \& Kalakanis, D. (2000). Differences in control of limb dynamics during dominant and nondominant arm reaching. Journal of Neurophysiology, 83(5), 2661-2675.

Sainburg, R. L. (2002). Evidence for a dynamic-dominance hypothesis of handedness. Experimental brain research, 142(2), 241-258. https://doi.org/10.1007/s00221-001-0913-8

Sainburg, R. L. (2005). Handedness: differential specializations for control of trajectory and position. Exercise and sport sciences reviews, 33(4), 206-213.

Sainburg, R. L., \& Schaefer, S. Y. (2004). Interlimb differences in control of movement extent. Journal of Neurophysiology, 92(3), 1374-1383. https://doi.org/10.1152/jn.00181.2004

Sainburg, R. L., Schaefer, S. Y., \& Yadav, V. (2016). Lateralized motor control processes determine asymmetry of interlimb transfer. Neuroscience, 334, 26-38. https://doi.org/10.1016/j.neuroscience.2016.07.043

Saucedo Marquez, C. M., Zhang, X., Swinnen, S. P., Meesen, R., \& Wenderoth, N. (2013). Task-specific effect of transcranial direct current stimulation on motor learning. Frontiers in human neuroscience, 7, 333. https://doi.org/10.3389/fnhum.2013.00333

Schaefer, S. Y., Haaland, K. Y., \& Sainburg, R. L. (2007). Ipsilesional motor deficits following stroke reflect hemispheric specializations for movement control. Brain, 130(8), 2146-2158. https://doi.org/10.1093/brain/awm145

Schaefer, S. Y., Haaland, K. Y., \& Sainburg, R. L. (2009). Dissociation of initial trajectory and final position errors during visuomotor adaptation following unilateral stroke. Brain research, 1298, 78-91. https://doi.org/10.1016/j.brainres.2009.08.063

Schaefer, S. Y., Mutha, P. K., Haaland, K. Y., \& Sainburg, R. L. (2012). Hemispheric specialization for movement control produces dissociable differences in online corrections after stroke. Cerebral Cortex, 22(6), 1407-1419. https://doi.org/10.1093/cercor/bhr237

Schambra, H. M., Abe, M., Luckenbaugh, D. A., Reis, J., Krakauer, J. W., \& Cohen, L. G. (2011). Probing for hemispheric specialization for motor skill learning: a transcranial direct current stimulation study. Journal of Neurophysiology, 106(2), 652-661. https://doi.org/10.1152/jn.00210.2011

Serrien, D. J., Ivry, R. B., \& Swinnen, S. P. (2006). Dynamics of hemispheric specialization and integration in the context of motor control. Nature Reviews Neuroscience, 7(2), 160-166. https://doi.org/10.1038/nrn1849

Shadmehr, R., \& Holcomb, H. H. (1997). Neural correlates of motor memory consolidation. Science, 277(5327), 821-825. https://doi.org/ 10.1126/science.277.5327.821

Stagg, C. J., Best, J. G., Stephenson, M. C., O'Shea, J., Wylezinska, M., Kincses, Z. T., . . Johansen-Berg, H. (2009). Polarity-sensitive modulation of cortical neurotransmitters by transcranial stimulation. The Journal of Neuroscience, 29(16), 5202-5206. https://doi.org/ 10.1523/JNEUROSCI.4432-08.2009

Stöckel, T., \& Wang, J. (2011). Transfer of short-term motor learning across the lower limbs as a function of task conception and practice order. Brain and cognition, 77(2), 271-279. 
https://doi.org/10.1016/j.bandc.2011.07.010

Stöckel, T., \& Weigelt, M. (2012). Brain lateralisation and motor learning: Selective effects of dominant and non-dominant hand practice on the early acquisition of throwing skills. Laterality: Asymmetries of Body, Brain and Cognition, 17(1), 18-37. https://doi.org/0.1080/1357650X.2010.524222

Utz, K. S., Dimova, V., Oppenländer, K., \& Kerkhoff, G. (2010). Electrified minds: transcranial direct current stimulation (tDCS) and galvanic vestibular stimulation (GVS) as methods of non-invasive brain stimulation in neuropsychology — a review of current data and future implications. Neuropsychologia, 48(10), 2789-2810. https://doi.org/10.1016/j.neuropsychologia.2010.06.002

Vines, B. W., Cerruti, C., \& Schlaug, G. (2008). Dual-hemisphere tDCS facilitates greater improvements for healthy subjects' non-dominant hand compared to uni-hemisphere stimulation. BMC neuroscience, 9(1), 103. https://doi.org/10.1186/1471-2202-9-103

Vines, B. W., Nair, D., \& Schlaug, G. (2008). Modulating activity in the motor cortex affects performance for the two hands differently depending upon which hemisphere is stimulated. European Journal of Neuroscience, 28(8), 1667-1673. https://doi.org/10.1111/j.1460-9568.2008.06459.x

Wulf, G., \& Schmidt, R. A. (1994). Feedback-induced variability and the learning of generalized motor programs. Journal of Motor Behavior, 26(4), 348-361. https://doi.org/10.1080/00222895.1994.9941691

Yadav, V., \& Sainburg, R. (2011). Motor lateralization is characterized by a serial hybrid control scheme. Neuroscience, 196, 153-167. https://doi.org/10.1016/j.neuroscience.2011.08.039

\section{Copyrights}

Copyright for this article is retained by the author(s), with first publication rights granted to the journal.

This is an open-access article distributed under the terms and conditions of the Creative Commons Attribution license (http://creativecommons.org/licenses/by/4.0/). 